\title{
Factores asociados a falla en el trasplante de córnea
}

\section{Factors associated with corneal transplant failure}

\author{
Gilda M. Vidaurrazaga-Sosa ${ }^{1}$, Karla D. Bravo-Ramírez y José M. Ornelas-Aguirre2*
}

${ }^{1}$ Servicio de Oftalmología, Centro Médico Nacional del Noroeste, Instituto Mexicano del Seguro Social; ${ }^{2}$ Departamento de Ciencias de la Salud, Universidad de Sonora. Ciudad Obregón, Sonora, México

\section{Resumen}

Objetivo: Analizar los factores clínicos antes y después de la cirugía asociados al rechazo de córnea. Método: Estudio descriptivo, transversal y analítico de 130 trasplantes de córnea en los que se estudiaron factores clínicos antes y después de la cirugía. Se incluyeron enfermos de ambos sexos y cualquier edad, con un seguimiento mínimo de 8 meses, excluyéndose procedimientos tectónicos. Se realizó un análisis descriptivo e inferencial para las variables sexo, diagnósticos previos, vascularización, tamaño de trépano, comorbilidad y antecedentes clínicos. Se consideró como significativo un valor de $p \leq 0.05$. Resultados: Se encontró un 31\% de rechazo. Se observó un mayor riesgo de rechazo en mujeres, en caso de trasplante de córnea previo, neovascularización en córnea receptora y enfermos con glaucoma. Conclusiones: Es necesario proyectar nuevos estudios prospectivos y de seguimiento que permitan evaluar de forma certera y estandarizada la evolución clínica de los pacientes que reciben un trasplante de córnea.

Palabras clave: Trasplante de córnea. Queratoplastia penetrante. Factores de riesgo. Rechazo.

\section{Abstract}

Purpose: To analyze the clinical factors associated with corneal rejection before and after surgery. Method: In a descriptive, cross-sectional and analytical study in 130 corneal transplants, clinical factors were studied before and after surgery. Patients of both sexes, and any age were included, with a minimum follow-up of 8 months, and tectonic procedures were excluded. A descriptive and inferential analysis was conducted for the variables of gender, previous diagnoses, vascularization, trepan size, comorbidities and past medical history. $P$ values $\leq 0.05$ were considered significant. Results: $A$ 31\% rejection rate was found. A higher risk of rejection was seen in women, previous corneal transplantation or neovascularization in the recipient's cornea and patients with glaucoma. Conclusion: It is necessary to plan new prospective and follow-up studies to assess accurately, and in a standard way, the clinical evolution of the patient who receives a cornea transplant.

Keywords: Corneal transplant. Penetrating keratoplasty. Risk factors. Rejection.

Correspondencia:

*José M. Ornelas-Aguirre

Paseo del Jardín 2106

Fracc. El Paraíso,

Fecha de recepción: 27-04-2021

C.P. 85039, Ciudad Obregón, Son., México

E-mail: manuel.ornelas@unison.mx
Disponible en internet: 03-01-2022 Rev Mex Oftalmol. 2022;96(1):22-26

www.rmo.com.mx 0187-4519/○ 2021 Sociedad Mexicana de Oftalmología. Publicado por Permanyer. Este es un artículo open access bajo la licencia CC BY-NC-ND (http://creativecommons.org/licenses/by-nc-nd/4.0/). 


\section{Introducción}

El trasplante de córnea es un procedimiento quirúrgico que lleva más de 100 años realizándose en todo el mundo ${ }^{1}$. Se lleva a cabo con elevada frecuencia y con una tasa de éxito excelente en comparación con otros tipos de trasplante de órganos. A pesar del privilegio inmunitario ${ }^{2,3}$ y la falta de vascularidad de la córnea, el rechazo sigue siendo la principal causa de falla en aquellos casos en que no se logra el éxito quirúrgico4-8.

Se estima que se realizan más de 60,000 trasplantes de córnea cada año $0^{9,10}$. De ellos, más del $30 \%$ de los pacientes experimentarán un episodio de rechazo y un $5-7 \%$ terminarán en falla del injerto $2,10,11$. La incidencia de rechazo reportada en varios estudios se encuentra entre el $2 \%$ y el $68 \% 2$.

Diversos estudios han identificado factores de riesgo del donador, del receptor e incluso intraoperatorios que influyen en la aparición de rechazo corneal; por tal motivo, es indispensable su evaluación previa a la queratoplastia. Se han estudiado extensamente la respuesta mediada por células $\mathrm{T}$ CD4+, los procesos inflamatorios y el trauma quirúrgico como factores que inducen el crecimiento vascular dentro de la córnea ${ }^{2,12}$. El rechazo rara vez ocurre dentro de las primeras 2 semanas tras el trasplante, y puede presentarse hasta 20 años después ${ }^{13}$.

Dentro de las causas primarias se han mencionado los trastornos inmunitarios y la falla celular endotelial que muy frecuentemente se asocia en el donador a la presencia de enfermedad endotelial primaria, edad avanzada, muerte traumática, uso de una técnica inadecuada para obtención del injerto o tiempo de preservación prolongado ${ }^{4,14}$. Se reconoce como principal causa secundaria no inmunitaria la falla endotelial tardía ${ }^{15}$.

En México, los primeros informes de trasplantes de córnea aparecen en la década de $1950^{2}$. Los factores quirúrgicos identificados en el pronóstico del injerto de córnea son el tamaño del injerto, la localización y las cirugías asociadas en el mismo acto quirúrgico (cataratas, iris, vítreo o retina) $)^{9,16}$. Los datos estadísticos actuales indican que la supervivencia del injerto corneal a 10 años se encuentra entre $65 \%$ y $95 \%{ }^{5}$. Sin embargo, esta cifra puede verse disminuida si el paciente presenta alguna otra enfermedad ocular (distrofia estromal-endotelial $55 \%$, leucoma corneal $49 \%$, trauma $33 \%$, heridas químicas $14 \%)^{5,6,17}$. Dentro de la comorbilidad asociada a falla en el injerto se incluyen la presencia de cataratas, errores de refracción y disfunción retiniana ${ }^{9}$.

El objetivo de este estudio fue analizar los factores de riesgo que se asocian significativamente con el rechazo del trasplante de córnea en un centro de referencia del noroeste de México.

\section{Método}

Estudio descriptivo de tipo transversal analítico en el que se estudiaron 130 individuos que fueron sometidos a trasplante de córnea en el Centro Médico Nacional del Noroeste del Instituto Mexicano del Seguro Social, en ciudad Obregón, Sonora. Las variables de estudio incluyeron el sexo, la edad, la vascularidad del injerto, el tamaño de trépano utilizado, la comorbilidad, el antecedente de glaucoma, la cirugía intraocular previa, la agudeza visual, el tiempo de rechazo, la cirugía combinada, el trasplante de córnea previo y el diagnóstico preoperatorio. Todos los participantes tenían un seguimiento mínimo de 8 meses posterior a la realización de la cirugía. Se excluyeron aquellos casos sometidos a queratoplastia penetrante, que carecían de diagnóstico preoperatorio o que no tuvieron seguimiento posterior al trasplante. El proyecto de investigación fue aprobado por el Comité Local de Investigación (R-2020-2602013). Toda la información se obtuvo a partir de los registros clínicos disponibles en la coordinación de trasplantes del hospital de enero de 2017 a diciembre de 2018. La información estadística fue procesada con ayuda del paquete estadístico SPSS versión 21.0 para Windows. Para el análisis estadístico se realizó una evaluación descriptiva de los datos con medidas de tendencia central y dispersión. El análisis inferencial de los factores de riesgo se realizó con tablas de contingencia mediante la prueba de $\chi^{2}$ de Pearson con cálculo de los intervalos de confianza al 95\% (IC95\%). Se realizó un análisis de riesgo mediante odds ratio (OR) para evaluar los factores que intervinieron en la falla del injerto. Las variables numéricas y de razón se estudiaron con ayuda de la prueba t de Student. Se consideró como estadísticamente significativo un valor de $p \leq$ 0.05 .

\section{Resultados}

Se realizó un estudio con 130 pacientes operados de queratoplastia penetrante, con edades entre 15 y 88 años, con un promedio de $56 \pm 19$ años. Cuarenta casos (31\%; IC95\%: 23-38) presentaron rechazo corneal. El rechazo ocurrió con más frecuencia en las mujeres (21 casos, $16 \%$ ). El tiempo promedio para establecerse el rechazo fue de 8 meses. De los 40 casos que presentaron rechazo, $33 \%$ fue en el tercer trimestre y $13 \%$ después de 12 meses del procedimiento quirúrgico. 
El diagnóstico preoperatorio más común fue la queratopatía bullosa en 56 pacientes (43\%; IC95\%: 34-51), seguido de leucoma corneal en 32 pacientes $(25 \%$; IC95\%: 17-32) y queratocono en 20 pacientes (15\%; IC95\%: 9-21). Las características de la población de estudio previo a la realización del trasplante de córnea se describen en la tabla 1. La comparación entre la agudeza visual antes y a los 12 meses del procedimiento quirúrgico mostró una mejoría en 69 pacientes (53\%; IC95\%: 44-61), empeoramiento en 20 pacientes (15\%; IC95\%: 9-21) y sin cambios en 41 pacientes (32\%; IC95\%: 23-39). Los antecedentes clínicos y comorbilidad en los pacientes intervenidos de trasplante de córnea se describen en la tabla 2.

Dentro de los factores que presentaron asociación con un mayor riesgo de rechazo del trasplante de córnea se encontraron el sexo femenino (OR: 1.8; $p=0.02$ ), el antecedente de trasplante de córnea (OR: $2.6 ; p=0.0001$ ), la neovascularización en la córnea receptora (OR: 2.0; $p$ $=0.004$ ), la neovascularización en los cuatro cuadrantes $(\mathrm{OR}=2.5 ; \mathrm{p}=0.001)$ y la presencia de glaucoma (OR: 2.2; $p=0.002$ ). Dentro del diagnóstico preoperatorio, se encontró que la presencia de queratocono es un factor protector para la falla del trasplante corneal (OR: 0.28; $p$ $=0.02$ ). Los factores de riesgo asociados a rechazo en trasplante de córnea se describen en la tabla 3.

\section{Discusión}

Este estudio es el primero que analiza el rechazo en el trasplante de córnea en el noroeste de México. Se halló una frecuencia de rechazo del $31 \%$, que se corresponde con lo reportado en estudios similares. SánchezCornejo, et al. ${ }^{13}$, en 110 casos de trasplante, tuvieron una tasa de rechazo del $52 \%$, mientras que en estudios más recientes, como el de Verdiguel-Sotelo, et al. ${ }^{2}$, en una cohorte de 539 pacientes, reportan un rechazo del $12 \%$, y Gittins-Núñez, et al. ${ }^{18}$, en 150 pacientes intervenidos, tuvieron un rechazo del $35 \%$. En todos estos estudios se observa una variación muy amplia para el rechazo y quizá esto se deba a su consistencia interna y al tamaño de muestra incluida para el análisis.

Diversos son los factores que se han sugerido para la aparición de rechazo corneal, como el tamaño del injerto, la vascularización por número de cuadrantes antes y después del trasplante, el tipo de sutura, la edad del paciente, la presencia de trasplantes previos 0 tener patologías asociadas, como glaucoma ${ }^{13}$. El rechazo raramente aparece en las primeras 2 semanas y puede producirse hasta años después de una queratoplastia penetrante ${ }^{19}$.
Tabla 1. Características de la población de estudio previamente a la realización del trasplante de córnea

\begin{tabular}{|l|c|c|c|c|c|}
\hline & $n$ & $\%$ & IC95\% & OR & P \\
\hline $\begin{array}{l}\text { Edad } \\
\quad 40 \text { años }\end{array}$ & 31 & 24 & $16-31$ & & \\
\hline $\begin{array}{l}40-60 \text { años } \\
>60 \text { años }\end{array}$ & 27 & 21 & $14-28$ & & \\
\hline $\begin{array}{l}\text { Diagnóstico previo } \\
\text { Queratopatía bullosa }\end{array}$ & 52 & 55 & $46-63$ & & \\
\hline $\begin{array}{l}\text { Queratocono } \\
\text { Leucoma }\end{array}$ & 20 & 43 & $34-51$ & 1.0 & 0.76 \\
\hline $\begin{array}{l}\text { Rechazo de injerto } \\
\text { corneal }\end{array}$ & 32 & 25 & $17-32$ & 0.7 & 0.02 \\
\hline $\begin{array}{l}\text { Distrofia de Fuch } \\
\text { Úlcera corneal }\end{array}$ & 12 & 9 & $4-14$ & 1.0 & 0.41 \\
\hline
\end{tabular}

IC95\%: intervalo de confianza al 95\%; OR: odds ratio.

Fuente: registros clínicos del servicio de oftalmología del Centro Médico Nacional del Noroeste en Ciudad Obregón, Sonora, México.

Tabla 2. Antecedentes clínicos y comorbilidad en los pacientes intervenidos de trasplante de córnea

\begin{tabular}{|c|c|c|c|c|c|}
\hline & n & $\%$ & IC95\% & OR & $\mathbf{p}$ \\
\hline \multicolumn{6}{|l|}{ Ojo intervenido } \\
\hline Derecho & 58 & 45 & $38-55$ & & \\
\hline Izquierdo & 71 & 55 & $46-63$ & & \\
\hline \multicolumn{6}{|l|}{ Antecedentes } \\
\hline prequirúrgicos & 18 & 14 & $8-19$ & 2.6 & 0.0001 \\
\hline Trasplante de córnea & 64 & 49 & $40-57$ & 1.2 & 0.38 \\
\hline Cirugía intraocular & 48 & 37 & $28-45$ & 2.0 & 0.004 \\
\hline $\begin{array}{l}\text { Neovascularización en } \\
\text { córnea receptora }\end{array}$ & & & & & \\
\hline \multicolumn{6}{|l|}{ Comorbilidad } \\
\hline Diabetes mellitus & 29 & 23 & $14-27$ & 1.4 & 0.20 \\
\hline Hipertensión arterial & 55 & 42 & $34-51$ & 1.5 & 0.11 \\
\hline Enfermedad reumatoide & 4 & 3 & $0.1-6$ & 0.8 & 0.80 \\
\hline Hipotiroidismo & 7 & 5 & $1-9$ & 0.9 & 0.80 \\
\hline Glaucoma & 32 & 25 & $17-32$ & 2.2 & 0.002 \\
\hline
\end{tabular}

IC95\%: intervalo de confianza al 95\%; 0R: odds ratio.

Fuente: registros clínicos del servicio de oftalmología del Centro Médico Nacional del Noroeste en Ciudad Obregón, Sonora, México.

En nuestra serie, la agudeza visual a los 12 meses del trasplante mejoró en más de la mitad de los casos, pero fue inferior a lo reportado por Gittins-Núñez, et al. ${ }^{18}$, quienes hallaron una mejoría hasta del $81 \%$. Dentro de los factores que influyeron en el éxito de la agudeza visual en nuestro estudio se encuentran la presencia de glaucoma con daño al nervio óptico, la retinopatía diabética preexistente y la hemorragia vítrea, entre otros. Siempre será recomendable el estudio de estos antecedentes para un buen éxito posterior a la cirugía. 
Tabla 3. Factores de riesgo asociados a rechazo en trasplante de córnea

\begin{tabular}{|c|c|c|c|c|}
\hline & Sin rechazo $(n=90)$ & Con rechazo $(\mathrm{n}=40)$ & OR & p \\
\hline $\begin{array}{l}\text { Sexo } \\
\text { Femenino } \\
\text { Masculino }\end{array}$ & $\begin{array}{l}28(21.5 \%) \\
62(47.7 \%)\end{array}$ & $\begin{array}{l}21(16.2 \%) \\
19(14.6 \%)\end{array}$ & 1.8 & 0.02 \\
\hline $\begin{array}{l}\text { Antecedentes } \\
\text { Trasplante de córnea } \\
\text { Cirugía intraocular } \\
\text { Neovascularización en córnea receptora }\end{array}$ & $\begin{array}{c}6(4.6 \%) \\
42(32.3 \%) \\
26(20.0 \%)\end{array}$ & $\begin{array}{c}12(9.2 \%) \\
22(16.9 \%) \\
22(16.9 \%)\end{array}$ & $\begin{array}{l}2.6 \\
1.2 \\
2.0\end{array}$ & $\begin{array}{l}0.0001 \\
0.380 \\
0.004\end{array}$ \\
\hline $\begin{array}{l}\text { Comorbilidad } \\
\text { Diabetes mellitus } \\
\text { Hipertensión arterial } \\
\text { Enfermedad reumática } \\
\text { Hipotiroidismo } \\
\text { Glaucoma } \\
\text { Cirugía combinada }\end{array}$ & $\begin{array}{c}17(12.3 \%) \\
34(26.2 \%) \\
3(2.3 \%) \\
5(3.8 \%) \\
15(11.5 \%) \\
13(10 \%)\end{array}$ & $\begin{array}{c}12(8.5 \%) \\
21(16.2 \%) \\
1(0.8 \%) \\
2(1.5 \%) \\
17(13.1 \%) \\
7(5.4 \%)\end{array}$ & $\begin{array}{l}1.4 \\
1.5 \\
0.8 \\
0.9 \\
2.2 \\
1.1\end{array}$ & $\begin{array}{c}0.207 \\
0.117 \\
0.8 \\
0.8 \\
0.002 \\
0.65\end{array}$ \\
\hline $\begin{array}{l}\text { Tamaño del trépano } \\
<7 \\
7.25-7.5 \\
7.75-8.0\end{array}$ & $\begin{array}{c}2(1.5 \%) \\
67(51.5 \%) \\
21(16.2 \%)\end{array}$ & $\begin{array}{c}1(0.8 \%) \\
27(20.8 \%) \\
12(9.2 \%)\end{array}$ & $\begin{array}{l}1.0 \\
0.7 \\
1.2\end{array}$ & $\begin{array}{l}0.92 \\
0.41 \\
0.42\end{array}$ \\
\hline
\end{tabular}

OR: odds ratio.

Fuente: registros clínicos del servicio de oftalmología del Centro Médico Nacional del Noroeste en Ciudad Obregón, Sonora, México.

La causa más frecuente para la realización de trasplante de córnea fue la queratopatía bullosa, y en segundo lugar el queratocono, dato similar a lo reportado en la serie de Gittins-Núñez, et al. ${ }^{18}$, y posiblemente explicado por la edad de nuestra población, ya que la mayoría tenían 60 años o más y en muchos de ellos existía el antecedente de cirugía de catarata. Con respecto al sexo, en la literatura revisada no se presentan asociaciones entre este y el riesgo de rechazo ${ }^{14}$.

Dentro de los factores asociados al rechazo temprano del trasplante encontramos como más frecuentes el sexo femenino, el trasplante de córnea previo, la presencia de neovascularización en la córnea receptora y la neovascularización en los cuatro cuadrantes, así como la presencia de glaucoma (antes ya comentado). De todos estos factores, la vascularización corneal es muy relevante, pues representa la respuesta a un fenómeno agresor que modifica las características de la córnea, como inflamación, cicatrización o isquemia. De hecho, la presencia de vasos en la periferia corneal, en los cuatro cuadrantes, aumenta hasta nueve veces la probabilidad de que se presente rechazo del injerto ${ }^{13}$. Por ello, es necesario estandarizar la medición preoperatoria de la neovascularización en los cuatro cuadrantes y así tomar las medidas necesarias para disminuir el rechazo.

Respecto al momento posterior al procedimiento quirúrgico en que ocurrió el episodio de rechazo corneal, el mayor número de casos se dio durante el tercer trimestre (33\%), lo cual difiere de lo reportado por otros estudios en los que el rechazo ocurrió de forma temprana durante el primer trimestre tras la cirugía ${ }^{18}$.

El antecedente de rechazo previo fue el factor preoperatorio que más se asoció con rechazo; en cambio, el queratocono se observó como factor protector.

Dentro de las limitantes de este estudio se puede mencionar el hecho de ser retrospectivo, la dependencia de las observaciones que fueron realizadas bajo criterio del evaluador en turno y que no permitió estandarizar la metodología de recolección de la información clínica; sin embargo, durante la recolección de la información para este estudio se desechó aquella que tuviera errores o inconsistencias para evitar en lo posible sesgos de interpretación o registro.

La cirugía de catarata es uno de los principales procedimientos quirúrgicos que se realizan en todo el mundo. El resultado final en el proceso de donación y trasplante depende de múltiples factores, desde las características epidemiológicas de los candidatos a ser donante o receptor, hasta los procedimientos que se inician en la obtención, la preparación del procedimiento y la conservación del tejido, lo cual obliga a transitar por una serie de eventos que, de no cumplir con las normas establecidas, ponen en riesgo el éxito del procedimiento quirúrgico ${ }^{19,20}$. Por esta razón, es necesario optimizar la técnica quirúrgica, así como realizar un seguimiento posquirúrgico estrecho de los pacientes intervenidos 
para brindar una atención oportuna a las complicaciones.

\section{Conclusiones}

Los resultados de nuestro estudio no distan de lo reportado en otros similares, tanto nacionales como internacionales. Es necesario proyectar nuevos estudios prospectivos y de seguimiento que permitan evaluar de forma certera y estandarizada la evolución clínica de los pacientes que reciben un trasplante de córnea.

\section{Financiamiento}

Los autores declaran que para esta investigación no se recibió financiamiento.

\section{Conflicto de intereses}

Los autores declaran que no existe conflicto de interés alguno en este estudio.

\section{Responsabilidades éticas}

Protección de personas y animales. Los autores declaran que los procedimientos seguidos se conformaron a las normas éticas del comité de experimentación humana responsable y de acuerdo con la Asociación Médica Mundial y la Declaración de Helsinki.

Confidencialidad de los datos. Los autores declaran que han seguido los protocolos de su centro de trabajo sobre la publicación de datos de pacientes.

Derecho a la privacidad y consentimiento informado. Los autores declaran que en este artículo no aparecen datos de pacientes.

\section{Bibliografía}

1. Barut Selver O, Karaca I, Palamar M, Egrilmez S, Yagci A. Graft failure and repeat penetrating keratoplasty. Exp Clin Transplant. 2021;19:72-6.

2. Verdiguel-Sotelo K, Carrasco-Quiroz A, Rangel-Servín J. Categoría pronóstica de trasplante corneal en un centro de referencia. Rev Med Inst Mex Seguro Soc. 2016;54:738-45.

3. van Essen TH, Roelen DL, Williams KA, Jager MJ. Matching for human leukocyte antigens (HLA) in corneal transplantation - to do or not to do. Prog Retin Eye Res. 2015;46:84-110.

4. Ziaei M, Sharif-Paghaleh E, Manzouri B. Pharmacotherapy of corneal transplantation. Expert Opin Pharmacother. 2012;13:829-40

5. Barraquer RI, Pareja-Aricò L, Gómez-Benlloch A, Michael R. Risk factors for graft failure after penetrating keratoplasty. Medicine (Baltimore). 2019;98:e15274.

6. García-Alcolea EE, Pérez-Tejeda A, Acuña-Pardo A. Consideraciones inmunológicas sobre el rechazo del trasplante de córnea. Rev Cuba Hematol Inmunol Hemoter. 2010;26:306-14.

7. Guilbert E, Laroche L, Borderie V. Le rejet d'allogreffe de cornée. J Fr Ophtalmol. 2011;34:331-48.

8. Tan DT, Dart JK, Holland EJ, Kinoshita S. Corneal transplantation. Lancet. 2012;379:1749-61.

9. Panda A, Vanathi M, Kumar A, Dash Y, Priya S. Corneal graft rejection. Surv Ophthalmol. 2007;52:375-96.

10. Coster DJ, Williams KA. The impact of corneal allograft rejection on the long-term outcome of corneal transplantation. Am J Ophthalmol. 2005; $140: 1112-22$

11. Rojas-Dosal JA. Trasplante de córnea. Rev Hosp Jua Mex. 2000;67:139-43.

12. Klebe S, Coster DJ, Williams KA. Rejection and acceptance of corneal allografts. Curr Opin Organ Transplant. 2009;14:4-9.

13. Sánchez-Cornejo M, Olivares-Morales OE, Lima-Gómez V, Razo-Blanco Hernández DM, Pérez-Taibo $\mathrm{C}$. Factores asociados con rechazo a trasplante de córnea por grupos de pronóstico. Rev Mex Oftalmol. 2008;82:277-80.

14. López-Hernández SM, Díaz-Castillo FM, Gómez-Castillo Z, Pérez-Parra Z, Fernández-García K, Guerra-Almaguer M. Rechazo corneal en pacientes operados de queratoplastia penetrante óptica. Rev Cubana Oftalmol. 2018;31:1-13.

15. Kharod-Dholakia B, Randleman JB, Bromley JG, Stulting RD. Prevention and treatment of corneal graft rejection: current practice patterns of the Cornea Society (2011). Cornea. 2015;34:609-14.

16. Consejo de Salubridad General. Guía de práctica clínica para la quera- toplastia penetrante del Instituto Mexicano del Seguro Social (IMSS-541-12).

17. Tan DT, Dart JK, Holland EJ, Kinoshita S. Corneal transplantation. Lancet. 2012;379:1749-61.

18. Gittins-Núñez LO, Huerta-Albañil I, Ríos-Prado R, Soto-Dávila MA. Factores de riesgo y frecuencia de rechazo en pacientes con queratoplastia penetrante. Rev Med Inst Mex Seguro Soc. 2016;54:190-5.

19. Maguire MG, Stark WJ, Gottsch JD, Stulting RD, Sugar A, Fink NE, et al. Risk factors for corneal graft failure and rejection in the collaborative corneal transplantation studies. Collaborative Corneal Transplantation Studies Research Group. Ophthalmology. 1994;101:1536-47.

20. Escalona-Leyva ET, Pérez-Parra Z, Castillo-Pérez A, Jareño-Ochoa M, López-Hernández SM. Perfil epidemiológico del donante y del receptor en trasplante de córnea en el Instituto Cubano de Oftalmología «Ramón Pando Ferrer». Rev Cuba Oftalmol. 2014;27:558-68. 